\title{
On the Long Run Implications of Repeated Moral Hazard
}

\author{
Christopher Phelan \\ J. L. Kellogg Graduate School of Management, Northwestern University, \\ 2001 Sheridan Road, Evanston, Illinois 60208 \\ E-mail: cphelan@nwu.edu
}

Received September 24, 1996; revised December 4, 1997

\begin{abstract}
This paper analyzes the effects of moral hazard on long-run consumption or utility. Given exponential utility, it is shown that the utility of those with unobservable endowments becomes arbitrarily negative as long as any positive fraction of otherwise identical agents have observable endowments. Next, it is shown that assuming a finite number of agents results in essentially the same outcomes as with a continuum of agents. Finally, it is shown that the key characteristic determining whether the utility of almost all agents becomes arbitrarily low is whether $\lim _{c \rightarrow \infty} U^{\prime}(c)$ is bounded away from zero. Journal of Economic Literature Classification Numbers: D30; D31; D80; D82. (C) 1998 Academic Press
\end{abstract}

\section{INTRODUCTION}

The repeated unobserved endowment economies of Green [4] and Thomas and Worrall [9] have provoked much interest not only because they are viewed as simple and natural environments for exploring limited insurance in a dynamic setting, but also because of the extreme results which they derive. In Green's model, the efficient societal arrangement regarding a continuum of agents associated with an incentive problem (unobservable endowments) involves their eventual "immiserization" (almost all agents' consumption diverging to negative infinity). The single agent model of Thomas and Worrall [9] (where the agent faces a single risk neutral principal) independently derives the same result more generally: For the class of utility functions considered, the agent's utility almost surely becomes arbitrarily negative. These results seemed to imply that there was something about repeated moral hazard which caused a downward trend in the consumption of agents associated with an incentive problem. Later papers seem to show that such an implication is false: By considering different sets of assumptions than those in [4] and [9], one can create environments where agents' consumption distributions continually spread without a negative drift (Atkeson and Lucas [2]), or the 
consumption distribution converges to a bounded limiting distribution (Wang [10]).

It is difficult to tell from this collection of papers which results represent fragile special cases and which results hold more generally. Further, it is difficult to see overall what the key characteristics of moral hazard environments are which determine the long run consumption of agents. This paper attempts to rectify this problem by isolating the roles of the assumptions in the above models. I argue that the effects of moral hazard on consumption in the long run has little to do with the technology determining resource constraints (a focus of Atkeson and Lucas [2]) or the number of agents (a focus of Wang [10]) but instead hinges, somewhat unappealingly, on characteristics of the utility function.

The first section isolates the role of resource constraints using the exponential utility specification of Green [4]. In [4] (as well as Thomas and Worrall [9]), society can shift consumption across dates using a technology which discounts at a rate corresponding to the utility rate of discount. I show here (restating a result of Atkeson and Lucas [2]) that perturbing the economy of [4] to require that aggregate consumption equal the aggregate endowment date by date implies that each agent's consumption is the sum of an i.i.d. term and a term which follows a random walk without drift, overturning the immiserization result. This would seem to imply that the assumption in [4] of an ability of society to transfer consumption across dates at a fixed interest rate equal to the utility rate of discount is solely responsible for the immiserization result and not the assumption of repeated moral hazard. I argue here that such an implication is false. I show that if one imposes a date by date resource constraint but allows any positive fraction of agents to have observable endowments, the consumption of almost all agents with unobservable endowments goes to negative infinity while the consumption of those agents with observable endowments goes to positive infinity.

The second section isolates the role of the number of agents. I show here that assuming a finite number of agents instead of a continuum essentially leaves unchanged the results of [4] regardless of whether there is a period by period or present value resource constraint. This is a very different result from Wang [10] who modifies [4] by additionally imposing a lower bound on consumption and utility. ${ }^{1}$ With this bounding of utilities, [10] proves the existence of a bounded limiting distribution of consumption with movement. Agents do not get "stuck" at any particular consumption level but forever move about the limiting distribution.

\footnotetext{
${ }^{1}$ The bounding of utilities in [10] is implicit. Wang [10] assumes utility is defined on the entire real line, with restrictions that $U^{\prime}(c)$ exist and be positive everywhere, and that consumption be non-negative. This implies that the utility is bounded below on the consumption set.
} 
The third section considers the role of the characteristics of the utility function when the rate of interest is set to the utility rate of discount. While the first two sections focus on exponential utility, this section considers what results are special to this utility specification and what generalizes. This is important because the results of Thomas and Worrall [9] imply the utility of agents converging to negative infinity for a seemingly very general set of utility functions. I dispute this by considering an example here which perturbs [4] (as well as [9]) only in the choice of utility function. (Further, this is a well-behaved function: strictly increasing, concave, with decreasing absolute risk aversion.) In this example, by varying endowments, one can deliver an arbitrarily high fraction of agents diverging to either positive or negative infinity. The key assumption which determines whether a positive mass of utility paths tends to positive infinity is whether marginal utility is bounded away from zero as consumption increases.

One type of assumption that is purposely not discussed here is the role of bounds on either consumption or utility. Phelan [7] discusses the role of endogenous bounds on continuation utility given limited commitment. I argue here that the results of [10] crucially depend on utility bounds only by showing how they are overturned by considering the no bound alternative. Further, the paper of Aiyagari and Alvarez [1] can be considered a study regarding when utility bounds in incentive models are reflecting or absorbing. The strategy of this paper is to only consider environments where the set of feasible continuation utilities is open, and thus isolate the role of moral hazard on long run consumption outcomes independent of how moral hazard may interact with commitment issues.

\section{THE ROLE OF RESOURCE CONSTRAINTS}

Consider a world with a continuum of infinitely lived agents with unit mass. The physical aspects of each period are identical. In each period, each agent receives an unobservable perishable endowment $e$. (Assume a finite number of possible realizations $\left\{e_{1}, \ldots, e_{M}\right\}$, with $e_{i}>e_{j}$ for $i>j$.) Let $\pi(e)$ denote the fraction of agents who receive a particular $e$ realization. The fraction $\pi(e)$ is also taken as the probability of any particular agent receiving endowment $e^{2} .^{2}$ Agents care about expected discounted utility where their single period utility function over consumption is assumed, as in [4], to be the constant absolute risk aversion specification $U\left(c_{t}\right)$ :

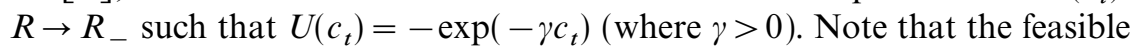
consumption set is assumed to be the entire real line. Agents discount future utils by the parameter $\beta<1$. These are identical assumptions as [4].

${ }^{2}$ Judd [5] shows that it is possible to choose a joint probability measure with these properties. 
It is easiest to consider the role of resource constraints if one posits (as in Phelan [6]) the existence of a price-taking financial intermediary or "representative firm" with which the agents sign enforceable lifetime contracts. This representative firm is assumed to have access a credit market which trades one-period ahead consumption in terms of current consumption. Let $\delta_{t}$ denote the price of $t+1$ period consumption in terms of date $t$ consumption. Given a specified sequence $\left\{\delta_{t}\right\}_{t=0}^{\infty}$, it is straightforward to show that a cost-minimizing firm can, without loss, treat each agent separately. Since endowments are assumed independent across agents, making the transfer to one agent contingent on the announced endowment of any other agent only lowers expected utility.

Consider the decision problem of a firm considering a contract for an agent promised a lifetime utility $w$. In this context, a "contract" specifies a possibly negative transfer from the firm to the agent at each date in the agent's life as a function of the history his endowment announcements. If one assumes $\delta_{t}=\delta<1$ for all $t$, (or the interest rate faced by the firm is constant) efficient contracts can be shown to be recursive with the expected discounted utility of the agent as the state variable.

Given the assumption of a constant $\delta$, the choice variables of the firm are as follows. For each lifetime utility level $w$, the firm chooses a transfer from the firm to the agent $T(e \mid w)$, and a function $W^{\prime}(e \mid w)$ specifying the agent's expected discounted utility from the beginning of the next period. The functions $T(e \mid w)$ and $W^{\prime}(e \mid w)$, along with the initial utility promise $w_{0}$, completely specify the lifetime contract.

This recursive formulation requires that the representative firm respect incentives and utility promises. For an agent who was promised a specific expected discounted utility $w$, a firm must pick policies which actually deliver this expected utility. This can be expressed in the form of the promise-keeping constraint (for each $w \in R_{-}$)

$$
w=\sum_{e} \pi(e)\left\{-\exp (-\gamma(e+T(e \mid w)))+\beta W^{\prime}(e \mid w)\right\} .
$$

The firm must also respect the unobservability of endowments. This is expressed as the incentive constraint (for each $w \in R_{-}$and $\{e, \hat{e}\}$ where $e>\hat{e})$

$$
\begin{aligned}
& -\exp (-\gamma(e+T(e \mid w)))+\beta W^{\prime}(e \mid w) \\
& \geqslant-\exp (-\gamma(e+T(\hat{e} \mid w)))+\beta W^{\prime}(\hat{e} \mid w) .
\end{aligned}
$$

This states that agents with high endowments must be willing to reveal them. (Note I assume that agents cannot over-report endowments.) 
Let $C^{*}(w)$ denote the minimized cost (or expected net transfers) in terms of current (or present value) consumption of providing expected discounted utility $w$, and $T^{*}(e \mid w)$ and $W^{*}(e \mid w)$ denote the policies which achieve $C^{*}(w)$. Now standard arguments imply $C^{*}(w)$ is a fixed point of the Bellman operator

$$
(B C)(w) \equiv \min _{T(e), W^{\prime}(e)} \sum_{e} \pi(e)\left(T(e)+\delta C\left(W^{\prime}(e)\right)\right),
$$

subject to (1) and (2), and that $T^{*}(e \mid w)$ and $W^{*}(e \mid w)$ solve the minimization in (3) for all $w$.

For the exponential utility case, Lemma 1 states that $T^{*}(e \mid w)$ and $W^{\prime *}(e \mid w)$ take a simple separable form. ${ }^{3}$

Lemma 1. If $U(c)=-\exp (-\gamma c)$, and $\delta<1$ is constant, the cost minimizing contract for the representative firm can be represented by functions $T^{*}(e \mid w)$ and $W^{*}(e \mid w)$ which take the separable form $T^{*}(e \mid w)=$ $-\log (-w) / \gamma+t(e), W^{\prime *}(e \mid w)=-w w^{\prime}(e)$.

\section{Proof. See Appendix.}

One can now examine the implications on consumption dynamics of the above result. If $w_{0}$ is an agent's initial utility and $e^{\tau}$ is his vector of his endowment realizations from $t=1 \cdots \tau$, his consumption at date $\tau$ is

$$
\begin{aligned}
c_{\tau} & =\frac{-\log \left(w_{0} \prod_{t=1}^{\tau-1}-w^{\prime}\left(e_{t}\right)\right)}{\gamma}+e_{\tau}+t\left(e_{\tau}\right) \\
& =\frac{-\log \left(-w_{0}\right)}{\gamma}+\sum_{t=1}^{\tau-1} \frac{-\log \left(-w^{\prime}\left(e_{t}\right)\right)}{\gamma}+e_{\tau}+t\left(e_{\tau}\right) .
\end{aligned}
$$

The simple arithmetic of logarithms guarantees that an agent's initial unconditional payment is permanent, as is every addition to it through the function $w^{\prime}(e)$. Each agent's consumption is the sum of an i.i.d. term (his endowment, $e_{\tau}$, plus his conditional transfer, $\left.t\left(e_{\tau}\right)\right)$ and a term (the remainder of Eq. (4)) which is a random walk (i.i.d. innovations).

Nowhere has a resource constraint been used. The above result is solely due to an assumed constant interest rate faced by the firm and the assumption of exponential utility. In Green [4], society (or the social planner) is

\footnotetext{
${ }^{3}$ This is not a new result. This result is proved by [4] and [9] for the case where $\delta=\beta$. Further, while stated differently, the result itself (for all $\delta$ ) is in Atkeson and Lucas [2, p. 444]. The proof here is different than in [2] and is useful as an outline of the strategy for the proofs of Lemmas 2 and 3 , which are new results.
} 
assumed able to transfer resources across dates at the utility rate of discount $\beta$. Thus the planner's problem in [4] is the firm's problem here with $\delta=\beta$. It happens to be at this particular $\delta$, the random walk component of consumption has a negative drift. (A discussion of why this occurs is in section four.) That consumption is the sum of random walk component and an i.i.d. component, however, holds for all $\delta<1$.

Now consider assuming a date by date resource constraint (as in Atkeson and Lucas [2]). A clear implication of this is that aggregate consumption must be equal at all dates since the aggregate endowment is equal at all dates. Since the above characterization holds for all $\delta<1$, if $\delta$ is chosen such that the random walk component of consumption is driftless, then aggregate consumption is indeed constant. Atkeson and Lucas [2] show this can be done. Further, Eq. (4) makes clear that initial utilities, $w_{0}$, can be chosen to scale up or down consumption at all dates such that this constant aggregate consumption equals the aggregate endowment. Let $\delta^{*}$ denote the value for $\delta$ such that aggregate consumption is constant. It is straightforward to show that $\delta^{*}<\beta$. (When $\delta=\beta$ the firm chooses a negative drift in consumption. Lowering the price of future consumption induces the firm to provide lifetime utils more in terms of future consumption.)

This would seem to imply that the assumption in [4] that $\delta=\beta$, and not moral hazard, is responsible for the negative drift in consumption since with the more "reasonable" $\delta^{*}$ derived from a closed economy, no such drift occurs. I argue with the following lemma that such an intuition is misleading.

Lemma 2. If $U(c)=-\exp (-\gamma c)$, aggregate consumption must equal the aggregate endowment date by date, and fraction $\mu \in(0,1)$ agents have observable endowments, efficiency implies almost all agents with unobservable endowments have consumption go to negative infinity, and all agents with observable endowments have consumption go to positive infinity.

Proof. Consider a representative firm which takes as given an ability to transfer consumption across dates at given prices. For now, assume a constant price $\delta$ of one-period ahead consumption. As when all agents have unobservable endowments, it is straightforward to then show that the price-taking firm can, without loss, treat each agent separately. Given this, the consumption characterization for agents with unobservable endowments is given by Eq. (4). Equation (4) implies that for these agents

$$
c_{\tau+1}-c_{\tau}=\frac{-\log \left(-w^{\prime}\left(e_{\tau}\right)\right)}{\gamma}+\left(e_{\tau+1}-e_{\tau}\right)+\left(t\left(e_{\tau+1}\right)-t\left(e_{\tau}\right)\right) .
$$

That $e$ is i.i.d. across agents and time implies that if one aggregates across all agents with unobservable endowments, the last two terms of (5) 
drop out and the first right-hand-side term becomes a constant, independent of $\tau$. Thus, for any given $\delta$, the aggregate consumption of those with unobservable endowments will equal the previous period's aggregate consumption for that group plus a constant.

Agents with observable endowments will have deterministic consumption paths due to the lack of an incentive problem. Cost minimization by the firm and exponential utility for the agents implies consumption in a given period is simply the previous period's consumption level plus a constant. This implies that over both groups, for any $\delta$, the representative firm will choose contracts which deliver aggregate consumption in a given period as the previous period's aggregate consumption plus a constant. Thus for these contracts to satisfy the resource constraint, one needs to find a $\delta$ which causes this constant addition, aggregated over both groups, to be zero.

If $\delta=\beta$ this constant addition is zero for those with observable endowments. On the other hand, the results of [4] and [9] (which assume $\delta=\beta$ ) imply that the consumption of those with unobservable endowments is the sum of an i.i.d. term and a term which follows a random walk with negative drift. This implies that the aggregate consumption of those with unobservable endowments is decreasing by a constant amount each date. Thus $\delta=\beta$ causes the firm to choose contracts where aggregate consumption decreases over time.

Next suppose $\delta=\delta^{*}<\beta$, where $\delta^{*}$ is the rate which causes the firm to choose $w^{\prime}(e)$ such that expectation of the right-hand side of (5) is zero. By definition, the mean consumption of those with unobservable endowments is now constant across dates. However, the consumption of those agents with observable endowments will now have a constant positive increase. Here, aggregate consumption increases over time.

For $\delta$ greater than $\delta^{*}$ and less than $\beta$, the consumption of those with unobservable endowments is a random walk with negative drift (plus an i.i.d. term), and the consumption of agents with observable endowments has a constant positive increase. Since the drift term for both types of agents is continuous in $\delta$, this implies there exists a $\delta$ such that $\delta^{*}<\delta<\beta$ where aggregate consumption is constant.

What is left to show is that the contract chosen by the representative firm given this $\delta$ is efficient. Suppose an efficient allocation delivers the same distribution of initial utilities as the contract chosen by the pricetaking representative firm, but where aggregate consumption is weakly less at all dates and strictly less at some date. Since the aggregate endowment is the same at all dates, efficiency requires that such an allocation not have one agent's consumption depend on the announced endowment of another. This implies the allocation could have been chosen by the firm and would have lowered its costs yielding a contradiction. 


\section{THE ROLE OF THE NUMBER OF AGENTS}

To contrast with the previous environment with a continuum of agents, consider a world with $N$ infinitely lived agents with names $n=1, \ldots, N$. Analogous to the previous environment, assume endowments are i.i.d. across both agents and time. Given this structure, the aggregate state of this economy is a vector $\mathbf{e}=\left(e^{1}, \ldots, e^{N}\right)$. Given the existence of aggregate uncertainty, let the price taking representative firm (as in Phelan [6]) have access to two sufficient spot markets: an insurance market which trades the consumption good contingent on the aggregate state in terms of non-contingent consumption, and a credit market which trades one-period ahead non-contingent consumption in terms of current state-contingent consumption. Let $B(e)$ denote the price of e-contingent consumption in terms of non-contingent consumption (thus $\sum_{\mathbf{e}} B(\mathbf{e})=1$ ) and $\delta(\mathbf{e})$ denote the price of one-period ahead non-contingent consumption in terms of current e-contingent consumption.

With a continuum of agents, we considered two cases: (1) where society could borrow and lend at the utility rate of discount, and (2) where aggregate consumption was constrained to equal the aggregate endowment date by date. The analogue here to the first case is where the representative firm faces prices $\delta(\mathbf{e})=\beta$ and $B(\mathbf{e})=\pi(\mathbf{e})$, (where $\pi(\mathbf{e})$ is the probability of endowment vector $\mathbf{e})$. Given this, the representative firm can treat each agent separately and the results are the same as for a continuum of agents. In the second case, prices $\delta(\mathbf{e})$ and $B(\mathbf{e})$ must cause the representative firm to set aggregate consumption equal to the aggregate endowment date by date.

Given the date by date resource constraint, the representative firm must now consider the joint contract for agents with required utilities, $\mathbf{w}=$ $\left(w^{1}, \ldots, w^{N}\right)$. Under the assumption that the price vectors $\delta(\mathbf{e})$ and $B(\mathbf{e})$ are the same for each date, efficient contracts can again be shown to be recursive. This allows the firm to consider the vector of expected discounted utilities of the agents as a state variable. Given this, the choice variables of the firm are for each vector $\mathbf{w}$, a transfer from the firm to each agent $\mathbf{T}(\mathbf{e} \mid \mathbf{w})=\left(T^{1}(\mathbf{e} \mid \mathbf{w}), \ldots, T^{N}(\mathbf{e} \mid \mathbf{w})\right)$, and a vector of functions $\mathbf{W}^{\prime}(\mathbf{e} \mid \mathbf{w})=$ $\left(W^{\prime 1}(\mathbf{e} \mid \mathbf{w}), \ldots, W^{\prime N}(\mathbf{e} \mid \mathbf{w})\right)$ specifying each agent's expected discounted utility from the beginning of the next period, for each vector of endowment announcements.

As before, there is an appropriate promise keeping condition (for each $w^{n} \in R_{-}$and each $n$ )

$$
w^{n}=\sum_{\mathbf{e}} \pi(\mathbf{e})\left\{-\exp \left(-\gamma\left(e^{n}+T^{n}(\mathbf{e} \mid \mathbf{w})\right)\right)+\beta W^{\prime n}(\mathbf{e} \mid \mathbf{w})\right\},
$$


and incentive condition (for each $n$ and $\left\{\mathbf{e},\left(\mathbf{e}^{-n}, \hat{e}^{n}\right)\right\}$ where $e^{n}>\hat{e}^{n}$ )

$$
\begin{aligned}
& -\exp \left(-\gamma\left(e^{n}+T^{n}(\mathbf{e} \mid \mathbf{w})\right)\right)+\beta W^{\prime n}(\mathbf{e} \mid \mathbf{w}) \\
& \quad \geqslant-\exp \left(-\gamma\left(e^{n}+T^{n}\left(\left(\mathbf{e}^{-n}, \hat{e}^{n}\right) \mid \mathbf{w}\right)\right)\right)+\beta W^{\prime n}\left(\left(\mathbf{e}^{-n}, \hat{e}^{n}\right) \mid \mathbf{w}\right) .
\end{aligned}
$$

Let $C^{*}(\mathbf{w}): \mathbf{R}_{-}^{n} \rightarrow \mathbf{R}$ denote the minimized cost in terms of current (or present value) non-contingent consumption of providing the $N$ agents with a vector of expected discounted utilities $\mathbf{w}$, and $\mathbf{T}^{*}(\mathbf{e} \mid \mathbf{w})$ and $\mathbf{W}^{\prime *}(\mathbf{e} \mid \mathbf{w})$ denote the policies which achieve $C^{*}(\mathbf{w})$. Analogous to the continuum agent economy, $C^{*}(\mathbf{w})$ is a fixed point of the Bellman operator

$$
(B C)(\mathbf{w}) \equiv \min _{\mathbf{T}(\mathbf{e} \mid \mathbf{w}), \mathbf{w}^{\prime}(\mathbf{e} \mid \mathbf{w})} \sum_{\mathbf{e}} B(\mathbf{e})\left\{\sum_{n} T^{n}(\mathbf{e} \mid \mathbf{w})+\delta(\mathbf{e}) C\left(\mathbf{W}^{\prime}(\mathbf{e} \mid \mathbf{w})\right)\right\},
$$

subject to (6) and (7), and $\mathbf{T}^{*}(\mathbf{e} \mid \mathbf{w})$ and $\mathbf{W}^{\prime *}(\mathbf{e} \mid \mathbf{w})$ solve the minimization in $(8)$ for all $\mathbf{w}$.

Lemma 3, analogous to Lemma 1, states that the efficient functions $\mathbf{T} *(\mathbf{e} \mid \mathbf{w})$ and $\mathbf{W}^{\prime *}(\mathbf{e} \mid \mathbf{w})$ take the same simple separable form.

Lemma 3. If $U(c)=-\exp (-\gamma c)$ and $\delta(\mathbf{e})$ and $B(\mathbf{e})$ are constant over time, the functions $\mathbf{T}^{*}(\mathbf{e} \mid \mathbf{w})$ and $\mathbf{W}^{* *}(\mathbf{e} \mid \mathbf{w})$ take the separable form $T^{n *}(\mathbf{e} \mid \mathbf{w})=-\log \left(-w^{n}\right) / \gamma+t^{n}(\mathbf{e}), W^{n *}(\mathbf{e} \mid \mathbf{w})=-w^{n} w^{\prime n}(\mathbf{e})$.

\section{Proof. See Appendix.}

One can now examine the implications on consumption dynamics for the case of a finite number of agents. If $\mathbf{w}_{0}$ is the vector of agent's initial utilities and $\left(\mathbf{e}_{1}, \ldots, \mathbf{e}_{\tau}\right)$ is the vector of endowment realizations from $t=1 \cdots \tau$, agent $n$ 's consumption at date $\tau$ is

$$
\begin{aligned}
c_{\tau}^{n} & =\frac{-\log \left(w_{0}^{n} \prod_{t=1}^{\tau-1}-w^{\prime n}\left(\mathbf{e}_{t}\right)\right)}{\gamma}+e_{\tau}^{n}+t^{n}\left(\mathbf{e}_{\tau}\right) \\
& =\frac{-\log \left(-w_{0}^{n}\right)}{\gamma}+\sum_{t=1}^{\tau-1} \frac{-\log \left(-w^{\prime n}\left(\mathbf{e}_{t}\right)\right)}{\gamma}+e_{\tau}^{n}+t^{n}\left(\mathbf{e}_{\tau}\right) .
\end{aligned}
$$

Again, the simple arithmetic of logarithms guarantees that an agent's initial unconditional payment is permanent, as is every addition to it through the function $w^{\prime n}(\mathbf{e})$. Each agent's consumption is the sum of an i.i.d. term (his endowment plus his conditional transfer $t^{n}$ ) and a term (the remainder of Eq. (9)) which is a random walk (i.i.d. innovations).

The resource constraint has not yet been used. The characterization above depends only on constant price vectors $\delta(\mathbf{e})$ and $B(\mathbf{e})$ and exponential utility. For aggregate transfers to be zero at each date and history, a 
vector of initial utilities $\mathbf{w}_{0}$ and prices $\delta(\mathbf{e})$ and $B(\mathbf{e})$ must be chosen correctly. Aggregate transfers on the first date are

$$
\sum_{n}\left(\frac{-\log \left(-w_{0}^{n}\right)}{\gamma}+t^{n}\left(\mathbf{e}_{1}\right)\right) .
$$

If aggregate transfers are to be the same (zero) regardless of the realization of $\mathbf{e}_{1}$, Eq. (10) makes clear that for all realization pairs $\left(\tilde{\mathbf{e}}_{1}, \hat{\mathbf{e}}_{1}\right)$

$$
\sum_{n} t^{n}\left(\tilde{\mathbf{e}}_{1}\right)=\sum_{n} t^{n}\left(\hat{\mathbf{e}}_{1}\right)
$$

On the second date, net transfers are

$$
\sum_{n}\left(\frac{-\log \left(-w_{0}^{n}\right)}{\gamma}+\frac{-\log \left(-w^{\prime n}\left(\mathbf{e}_{1}\right)\right)}{\gamma}+t^{n}\left(\mathbf{e}_{2}\right)\right) .
$$

Since net transfer must be the same (zero) for each date, comparing (12) with (10) (and using (11)) makes clear that a necessary condition for the resource constraint to be satisfied is for all $\mathbf{e}$,

$$
\sum_{n} \frac{-\log \left(-w^{\prime n}(\mathbf{e})\right)}{\gamma}=0 .
$$

(Note that conditions (11) and (13) are precisely the correct number of conditions to pin down the prices $B(\mathbf{e})$ and $\delta(\mathbf{e})$.) If Eq. (13) holds for each e realization, it certainly holds in expectation. Symmetry implies for any two agents $m$ and $n$

$$
\sum_{\mathbf{e}} \pi(\mathbf{e}) \frac{-\log \left(-w^{\prime m}(\mathbf{e})\right)}{\gamma}=\sum_{\mathbf{e}} \pi(\mathbf{e}) \frac{-\log \left(-w^{\prime n}(\mathbf{e})\right)}{\gamma} .
$$

Equation (14) and the expectation of (13) imply that for each agent $n$,

$$
\sum_{\mathbf{e}} \pi(\mathbf{e}) \frac{-\log \left(-w^{\prime n}(\mathbf{e})\right)}{\gamma}=0 .
$$

As with a continuum of agents and a date by date resource constraint, each agent's consumption is the sum of an i.i.d. term and a term which follows a driftless random walk.

Gaining an intuitive understanding what happens here is easier given a two-agent, two-endowment, equal initial utility example. In the first period, if both agents have the high endowment or both have the low endowment, no transfer occurs and the contract starts over. If one has a high and one has a low endowment, the agent with a high endowment transfers some 
consumption to the agent with the low endowment. In return, the agent with the low endowment makes a constant transfer in every subsequent period to the agent with the high endowment regardless of future endowments. Except for this constant unconditional transfer, the contract then starts over.

To restate, the assumption of a finite number of agents in no way changes the results for the case of exponential utility with no consumption bounds. If society can borrow and lend at the utility rate of discount $(\delta=\beta)$ and insure at prices corresponding to the probabilities, then with probability one, each agent's consumption diverges to negative infinity. If a date by date resource constraint is imposed, the consumption of each agent is the sum of an i.i.d. term and a term which follows a driftless random walk. These are the same results as with a continuum of agents.

This makes clear that the results of Wang [10] depend crucially on his assumption that $c \geqslant 0$. The effect of Wang's bounding of utilities is less clear. The case where agents have bounded consumption but unbounded utility (say, logarithmic utility) is ruled out by Wang's assumption that utility is increasing on the entire real line. If $\delta(\mathbf{e})=\beta$ and $B(\mathbf{e})=\pi(\mathbf{e})$, Thomas and Worrall [9] implies that each agent's consumption converges to zero with probability one. If there is a date by date resource constraint and a finite number of agents, whether the agents converge to the open utility boundaries or to a non-degenerate distribution is an open question.

\section{THE ROLE OF THE UTILITY FUNCTION}

This section considers the role of the utility function in determining longterm outcomes.

The role of the utility function can best be seen in these environments by assuming $\delta=\beta$ and extending the results of [9] which hold for a more general class of utility functions than exponential utility. Thomas and Worrall [9] show if $C(w)$ is the present-value cost to the firm of providing $w$ lifetime utils to an agent, then $C^{\prime}(w)$ follows a martingale as long as the set of feasible $w$ values is an open set. (This can be guaranteed if utility is unbounded below.) The argument is that one way to increase the agent's current lifetime utility by $\varepsilon$ utils (where $\varepsilon$ is small) is to promise him the incentive compatible continuation contract associated with an extra $\varepsilon / \beta$ lifetime utils starting tomorrow for each endowment realization today. If $\delta=\beta$, this has cost to the firm of $\sum_{e} \pi(e) C^{\prime}\left(w^{\prime}(e)\right)$. If tomorrow's lifetime utility levels $w^{\prime}(e)$ are interior, envelope arguments apply and this cost is also the marginal cost of providing utils today, $C^{\prime}(w)$, and thus $C^{\prime}(w)$ is a martingale. (This is where one needs the set of feasible continuation utilities 
to be open.) Thomas and Worrall [9] use the fact that non-negative martingales almost surely converge (Doob's martingale convergence theorem (see [3])) to show, for a more general class of utility functions than exponential, that almost all utility paths go to negative infinity.

Their argument is that efficiency requires that the agent's beginning of period continuation utility, $w$, must almost never converge to a point in the set of feasible continuation utilities. ${ }^{4}$ On the other hand, $C^{\prime}(w)$ must almost always converge, since it is a positive martingale. Since $C^{\prime}(w)$ is a strictly increasing function (from the strict convexity of $C(w)$-again, see [9]), this implies that $w$ must converge either to an open boundary of the set of feasible utilities or diverge to infinity or negative infinity in such a way as to make the limit of $C^{\prime}(w)$ finite. In the class of utility functions considered by [9], this implies $w$ converges to negative infinity. To better explain this result, I will follow [9] and examine two known functions which bound $C(w)$. Let $\underline{C}(w)$ denote the cost function associated with observable endowments, and $\bar{C}(w)$ denote the cost function associated with restricting the firm to making unconditional (and thus trivially incentive compatible) transfers. Figure 1 displays these functions for the exponential utility case. ${ }^{5}$

If $a$ denotes the lower bound on consumption (or negative infinity if consumption is unbounded), the assumptions $\lim _{c \rightarrow a} U(c)=-\infty$ and $\lim _{c \rightarrow a} U^{\prime}(c)=\infty$ imply $\lim _{w \rightarrow-\infty} \underline{C}^{\prime}(w)=\lim _{w \rightarrow-\infty} \vec{C}^{\prime}(w)=0$. This and convexity of $C(w)$ implies $\lim _{w \rightarrow-\infty} C^{\prime}(w)=0$, otherwise $C(w)$ would cross $\bar{C}(w)$ or $\underline{C}(w)$. Thus one way for $C^{\prime}(w)$ to converge is for $w \rightarrow-\infty$. Thomas and Worrall [9] further assume $\lim _{c \rightarrow \infty} U(c)<\infty$. This implies $\lim _{w \rightarrow b} C^{\prime}(w)=\lim _{w \rightarrow b} \bar{C}^{\prime}(w)=\infty$ where $b$ denotes the least upper bound on lifetime utility. If one imposes the weaker condition that $\lim _{c \rightarrow \infty}$ $U^{\prime}(c)=0$ (which allows $b=\infty$ ) it is still the case that $\lim _{w \rightarrow b} C^{\prime}(w)=$ $\lim _{w \rightarrow b} \bar{C}^{\prime}(w)=\infty$. This implies $C^{\prime}(w)$ is a strictly increasing function with $\lim _{w \rightarrow \infty} C^{\prime}(w)=\infty$. Thus the only way $C^{\prime}(w)$ can converge without $w$ converging to an interior point in the set of feasible utilities is for $w \rightarrow-\infty$.

I next argue that it is not the case that all reasonable utility functions cause utility to diverge only downward. In particular, if $\lim _{c \rightarrow \infty} U^{\prime}(c)>0$, then the utility of an arbitrarily high fraction of agents can approach positive infinity. I argue this somewhat informally by presenting an example.

Let consumption take on the entire real line and let $U(c)=$ $\alpha c-\exp (-\gamma c)$. This function displays strict concavity and strictly decreasing absolute risk aversion. It violates the assumptions of [9] only by being unbounded above. Further, the assumption that utility is bounded above is not used by [9] to show that $C^{\prime}(w)$ is strictly increasing or that efficiency

\footnotetext{
${ }^{4}$ Put simply, if $w$ converges, then consumption is no longer history dependent. This rules out insurance, which is inefficient.

${ }^{5}$ In the figure, $U(c)=-\exp (-c), e \in\{0,10\}$ with $\pi\left(e_{1}\right)=\pi\left(e_{2}\right)=1 / 2$ and $\beta=0.9$.
} 


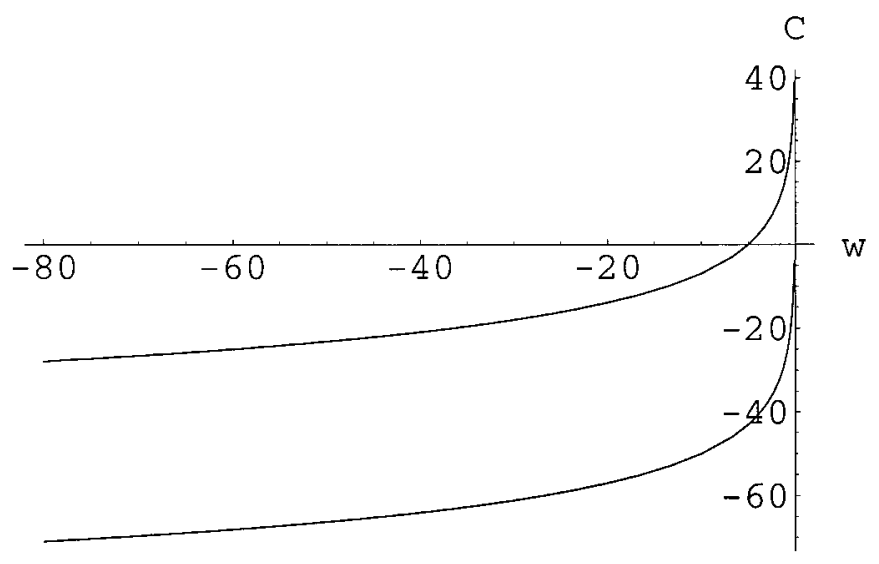

Fig. 1. $\bar{C}(w)$ and $\underline{C}(w)$ for exponential utility.

requires $w$ to almost never converge in the set of feasible utilities. The functions $\bar{C}(w)$ and $\underline{C}(w)$ for this case are displayed in Fig. 2 with $\alpha=\gamma=1$. As before, $\lim _{c \rightarrow-\infty} U^{\prime}(c)=\infty$ implies $\lim _{w \rightarrow-\infty} C^{\prime}(w)=\lim _{w \rightarrow-\infty} \bar{C}^{\prime}(w)=0$ and thus $\lim _{w \rightarrow-\infty} C^{\prime}(w)=0$. Thus it remains the case that one way for $C^{\prime}(w)$ to converge without $w$ converging to an interior point in the set of feasible utilities is for $w \rightarrow-\infty$. On the other hand, with $\lim _{c \rightarrow \infty} U^{\prime}(c)=$ $\alpha<\infty, \lim _{w \rightarrow \infty} \underline{C}^{\prime}(w)=\lim _{w \rightarrow \infty} \bar{C}^{\prime}(w)=1 / \alpha$ and thus $\lim _{w \rightarrow \infty} C^{\prime}(w)=$ $1 / \alpha$. Thus, in this example, another way for $C^{\prime}(w)$ to converge without $w$ converging to an interior point in the set of feasible utilities is for $w \rightarrow \infty$. Since $w$ must almost never converge in the set of feasible utilities, almost all agents must have $w \rightarrow \infty$ or $w \rightarrow-\infty$. One can next use properties of

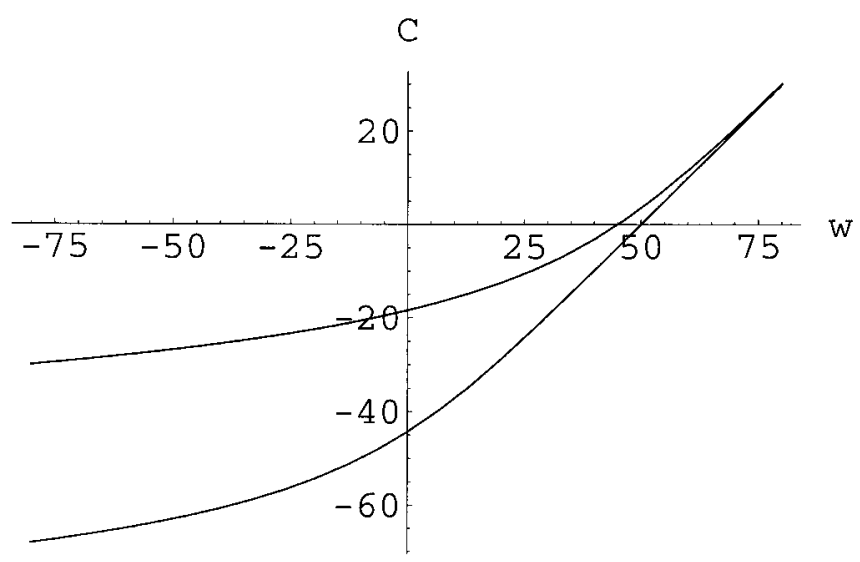

Fig. 2. $\bar{C}(w)$ and $\underline{C}(w)$ for exponential plus linear utility. 
$C^{\prime}(w)$ to show that the fraction of agents whose utility diverges upward is pinned down by initial conditions, and these conditions can be set to get an arbitrarily high or low fraction of agents go in either direction.

To do this, note that convexity of $C(w)$ implies $C^{\prime}(w)<1 / \alpha$ for all $w$. Thus unlike the case where $\lim _{c \rightarrow \infty} U^{\prime}(c)=0, C^{\prime}(w)$ is a bounded martingale. This implies not only that almost all $C^{\prime}(w)$ paths converge, but that the expected value of the limiting distribution of $C^{\prime}(w)$ equals the expected value of $C^{\prime}(w)$ at each date. Given this, it cannot be the case that almost all agents have $w \rightarrow-\infty$ or almost all agents have $w \rightarrow \infty$. Instead, an agent whose initial utility is $w_{0}$ must have $w \rightarrow \infty$ with probability $\alpha C^{\prime}\left(w_{0}\right)$ from the fact that $\lim _{w \rightarrow-\infty} C^{\prime}(w)=0$ and $\lim _{w \rightarrow \infty} C^{\prime}(w)=1 / \alpha$.

This implies that a particular agent's probability of diverging upward or downward is an increasing function only of his initial lifetime utility $w_{0}$. To pin this down, one must somehow determine initial lifetime utility promises. Suppose all agents are entitled to an initial lifetime utility $w_{0}$ such that $C\left(w_{0}\right)=0$, or the present expected value of the each agent's consumption stream equals the present expected value of his endowment stream. This is the initial utility which is delivered when agents initially own their endowments and there is competition in the provision of lifetime insurance contracts. Given this, one can set up endowments such that an arbitrarily high or low fraction of agents have $w \rightarrow \infty$.

To do this, consider some baseline values for the endowment levels $\left\{e_{1}, \ldots, e_{M}\right\}$ and probabilities $\pi(e)$ and consider adding an amount $x$ to each endowment level. Define $W_{A}(x)$ to be the utility of autarky (zero transfers at all states and dates) as a function of $x$, and $W_{F}(x)$ to be the utility of the full information contract (perfect insurance with zero expected transfers at each date) given $x$. Likewise, define $W(x)$ such that $C(W(x))=0$. Since $W_{A}(x)$ is unbounded above and $W(x) \geqslant W_{A}(x)$ (zero transfers is incentive compatible), $W(x)$ is unbounded above. This implies that $\lim _{x \rightarrow \infty} C^{\prime}(W(x))=1 / \alpha$ (because $\lim _{w \rightarrow \infty} C^{\prime}(w)=1 / \alpha$ ) or that as one increases $x$, the fraction of agents whose utility approaches infinity, $\alpha C^{\prime}(W(x))$, approaches unity. In the other direction, since $W_{F}(x)$ is unbounded below and $W(x) \leqslant W_{F}(x), W(x)$ is unbounded below. This implies $\lim _{x \rightarrow-\infty} C^{\prime}(W(x))=0$ (because $\lim _{w \rightarrow-\infty} C^{\prime}(w)=0$ ) or that as one decreases $x$, the fraction of agents whose utility approaches negative infinity, $1-\alpha C^{\prime}(W(x))$, approaches unity.

\section{CONCLUSIONS}

This paper analyzed the effects of moral hazard on long-run consumption or utility by isolating the effects of various types of assumptions. It argued that resource constraints and the number of agents were relatively 
unimportant while characteristics of the utility function were paramount. It was shown that date by date resource constraints fail to cause a zero trend in the expected consumption of agents facing moral hazard if any positive fraction of otherwise identical agents have observable endowments. Further, it is shown that if consumption is unbounded, the results for a continuum of agents go through for the case of a finite number of agents with or without a date by date resource constraint. Finally, it was shown that the key characteristic determining whether the consumption of almost all agents goes to its lower bound is whether $\lim _{c \rightarrow \infty} U^{\prime}(c)$ is bounded away from zero.

\section{APPENDIX}

Proof of Lemma 1. Consider a change of variables where $T(e \mid w)=$ $z(w)+t(e \mid w)$. Further, let $W^{\prime}(e \mid w)=\exp (-\gamma z(w)) w^{\prime}(e \mid w)$. This nonendowment-contingent payment $z(w)$ (and the scaling of utility promises by it) is simply harmless extra degrees of freedom in the firm's choice problem. For $z(w)$ set equal to any function, $t(e \mid w)$ and $w^{\prime}(e \mid w)$ can still be chosen to achieve any desired $T(e \mid w)$ and $W^{\prime}(e \mid w)$ functions.

Next, replace for $T(e \mid w)$ and $W^{\prime}(e \mid w)$ in the incentive condition (7) yielding a cancellation of $z(w)$, or,

$$
-\exp (-\gamma(e+t(e \mid w)))+\beta w^{\prime}(e \mid w) \geqslant-\exp (-\gamma(e+t(\hat{e} \mid w)))+\beta w^{\prime}(\hat{e} \mid w) .
$$

This implies that incentive compatibility is unaffected by the function $z(w)$.

Next, since it is harmless to set $z(w)$ to any function, let $z(w)=$ $-\log (-w) / \gamma$. Replacing for $T(e \mid w)$ and $W^{\prime}(e \mid w)$ in the promise keeping condition (6) yields the constraint,

$$
-1=\sum_{e} \pi(e)\left\{-\exp (-\gamma(e+t(e \mid w)))+\beta w^{\prime}(e \mid w)\right\} .
$$

This implies that under the assumption that $z(w)=-\log (-w) / \gamma$, the constraints for the firm can be written independent of the promised utility $w$. Next note that under this assumed $z(w)$, the function generating future utilities $W^{\prime}(e \mid w)$ can be written $W^{\prime}(e \mid w)=\exp (-\gamma z(w)) w^{\prime}(e \mid w)=-w$ $w^{\prime}(e \mid w)$.

Recall that $C^{*}(w)$ denotes the minimized cost (or expected net transfers) in terms of current (or present value) consumption of providing expected discounted utility $w$. Likewise, let $\underline{C}(w)$ denote the cost function associated with the full information problem and $\bar{C}(w)$ denote the cost function associated with restricting the firm to unconditional transfers. Since making 
transfers unconditional is incentive compatible, for all $w, \underline{C}(w) \leqslant C^{*}(w) \leqslant$ $\bar{C}(w)$. Further, $\bar{C}(w)-\underline{C}(w)$ can be shown to be bounded. (One way is to define $\widetilde{C}(w)$ as the cost of providing utility $w$ given that the lowest endowment occurs with probability 1 . Here, the contract subject to unconditional transfers, the full information contract, and the best incentive compatible contract are all the same. Further, it is clear that $\widetilde{C}(w) \geqslant \bar{C}(w)$. Finally, $\left.\widetilde{C}(w)-\underline{C}(w)=(1 /(1-\delta))\left(\sum_{e} \pi(e) e-e_{1}\right).\right)$

Given this, one can define a contraction operator $B$ on the space of functions between $\underline{C}(w)$ and $\bar{C}(w)$ with the supremum of the difference between functions as the metric. The cost function $C^{*}(w)$ is the unique fixed point of the contraction operator $B$ defined by

$$
(B C)(w) \equiv \min _{t(e \mid w), w^{\prime}(e \mid w) \in R_{-}}\left\{\frac{-\log (-w)}{\gamma}+t(e \mid w)+\delta C\left(-w w^{\prime}(e \mid w)\right)\right\}
$$

subject to (16) and (17).

One can guess and confirm that the function $C^{*}(w)$ takes the form

$$
C^{*}(w)=\frac{1}{1-\delta}\left(\frac{-\log (-w)}{\gamma}\right)+X
$$

as follows. First, if one substitutes the guessed form into (18) it becomes

$$
\begin{aligned}
(B C)(w) \equiv & \min _{t(e \mid w), w^{\prime}(e \mid w) \in R_{-}}\left\{\frac{1}{1-\delta} \frac{-\log (-w)}{\gamma}+t(e \mid w)\right. \\
& \left.+\delta\left(\frac{-\log \left(-w^{\prime}(e \mid w)\right)}{\gamma}+X\right)\right\} \\
= & \frac{1}{1-\delta} \frac{-\log (-w)}{\gamma} \\
& +\min _{t(e \mid w), w^{\prime}(e \mid w) \in R_{-}}\left\{t(e \mid w)+\delta\left(\frac{-\log \left(-w^{\prime}(e \mid w)\right)}{\gamma}+X\right)\right\}
\end{aligned}
$$

where each minimization is again subject to (16) and (17). Since the constraints are independent of $w$, the part of the objective function which depends on $w$ can be brought outside the minimimization problem. This shows $B$ preserves the guessed form, (which represents a compact subset of the space of functions lying between $\underline{C}(w)$ and $\bar{C}(w))$ thus confirming the guess form. Further, this shows that the functions which solve the minimization problem are independent of $w$. 
Proof of Lemma 3. This proof follows the same path as the proof to Lemma 1, differing only to take into account the joint choice of transfers and promises. Again consider a change of variables where $T^{n}(\mathbf{e} \mid \mathbf{w})=$ $z\left(w^{n}\right)+t^{\wedge}(\mathbf{e} \mid \mathbf{w})$ and let $W^{\prime n}(\mathbf{e} \mid \mathbf{w})=\exp \left(-\gamma z\left(w^{n}\right)\right) w^{\prime \wedge}(\mathbf{e} \mid \mathbf{w})$. As before, this non-endowment-contingent payment $z(w)$ (and the scaling of utility promises by it) is simply harmless extra degrees of freedom in the firm's choice problem. Again, replacing for $\mathbf{T}(\mathbf{e} \mid \mathbf{w})$ and $\mathbf{W}^{\prime}(\mathbf{e} \mid \mathbf{w})$ in the incentive condition (7) yields a cancellation of $z\left(w^{n}\right)$, or,

$$
\begin{aligned}
& -\exp \left(-\gamma\left(e^{n}+t^{n}(\mathbf{e} \mid \mathbf{w})\right)\right)+\beta w^{\prime n}(\mathbf{e} \mid \mathbf{w}) \\
& \quad \geqslant-\exp \left(-\gamma\left(e^{n}+t^{n}\left(\left(\mathbf{e}^{-n}, \hat{e}\right) \mid \mathbf{w}\right)\right)\right)+\beta w^{\prime n}\left(\left(\mathbf{e}^{-n}, \hat{e}\right) \mid \mathbf{w}\right),
\end{aligned}
$$

again implying that incentive compatibility is unaffected by the function $z\left(w^{n}\right)$.

Next, by setting $z\left(w^{n}\right)=-\log \left(-w^{n}\right) / \gamma$ and replacing for $\mathbf{T}(\mathbf{e} \mid \mathbf{w})$ and $\mathbf{W}^{\prime}(\mathbf{e} \mid \mathbf{w})$ in the promise keeping condition (6) yields the constraint,

$$
-1=\sum_{\mathbf{e}} \pi(\mathbf{e})\left\{-\exp \left(-\gamma\left(e^{n}+t^{n}(\mathbf{e} \mid \mathbf{w})\right)\right)+\beta w^{\prime n}(\mathbf{e} \mid \mathbf{w})\right\},
$$

again implying that under the assumption that $z\left(w^{n}\right)=-\log \left(-w^{n}\right) / \gamma$, the constraints for the firm can be written independent of the vector of promised utilities $\mathbf{w}$, and $W^{\prime n}(\mathbf{e} \mid \mathbf{w})=\exp \left(-\gamma z\left(w^{n}\right)\right) w^{\prime n}(\mathbf{e} \mid \mathbf{w})=-w^{n} w^{\prime n}(\mathbf{e} \mid \mathbf{w})$.

Let $C^{*}(\mathbf{w})$ denote the minimized cost (or expected net transfers) in terms of current non-contingent consumption of providing expected discounted utility vector $\mathbf{w}$, and note $C^{*}(\mathbf{w})$ is the fixed point of a contraction operator $B$ defined by

$$
\begin{aligned}
(B C)(\mathbf{w}) \equiv & \min _{\mathbf{t}(\mathbf{e} \mid \mathbf{w}), \mathbf{w}^{\prime}(\mathbf{e} \mid \mathbf{w})} \sum_{\mathbf{e}} B(\mathbf{e})\left\{\sum_{n}\left(\frac{-\log \left(-w^{n}\right)}{\gamma}+t^{n}(\mathbf{e} \mid \mathbf{w})\right)\right. \\
& \left.+\delta(\mathbf{e}) C\left(-w^{1} w^{1}(\mathbf{e} \mid \mathbf{w}), \ldots,-w^{N} w^{\prime N}(\mathbf{e} \mid \mathbf{w})\right)\right\}
\end{aligned}
$$

subject to (21) and (22).

Analogously to before, one can guess and confirm that the function $C^{*}(\mathbf{w})$ takes the form

$$
C^{*}(\mathbf{w})=\frac{1}{1-\bar{\delta}}\left(\sum_{n} \frac{-\log \left(-w^{n}\right)}{\gamma}\right)+X
$$

(where $\bar{\delta} \equiv \sum_{\mathbf{e}} B(\mathbf{e}) \delta(\mathbf{e})$ ) by substituting the guessed form into (23) and again noting that the mapping $B$ preserves the guessed form, and that the 
parts of the objective function which depend on $w$ can be brought outside the minimization problem.

\section{ACKNOWLEGMENTS}

The author thanks Fernando Alvarez, Andrew Atkeson, Narayana Kocherlakota, Ethan Ligon, Jonathan Thomas, two anonymous referees, and participants at seminars at Northwestern University and the Federal Reserve Bank of Richmond for helpful comments.

\section{REFERENCES}

1. R. Aiyagari and F. Alvarez, Stationary efficient distributions with private information and monitoring: A tale of kings and slaves, manuscript, Federal Reserve Bank of Minneapolis, 1995.

2. A. Atkeson and R. Lucas, On efficient distribution with private information, Rev. Econ. Stud. 59 (1992), 427-453.

3. P. Billingsley, "Probability and Measure," Wiley, New York, 1986.

4. E. Green, Lending and the smoothing of uninsurable income, in "Contractual Arrangements for Intertemporal Trade," Univ. of Minnesota Press, Minneapolis, 1987.

5. K. Judd, The 'law of large numbers' with a continuum of IID random variables, J. Econ. Theory 35 (1985), 19-25.

6. C. Phelan, Incentives and aggregate shocks, Rev. Econ. Stud. 61 (1994), 681-700.

7. C. Phelan, Repeated moral hazard and one-sided commitment, J. Econ. Theory 66(2) (1995), 488-506.

8. W. Rogerson, Repeated Moral Hazard, Econometrica 53 (1985), 69-76.

9. J. Thomas and T. Worrall, Income fluctuations and asymmetric information: An example of a repeated principal-agent problem, J. Econ. Theory 51 (1990), 367-390.

10. C. Wang, Dynamic insurance with private information and balanced budgets, Rev. Econ. Stud. 62(4) (1995), 577-596. 